\title{
A NOTE ON WITHDRAWAL FROM A TWO-LAYER FLUID THROUGH A LINE SINK IN A POROUS MEDIUM
}

\author{
G. C. HOCKING ${ }^{\bowtie 1}$ and H. ZHANG ${ }^{2}$ \\ (Received 23 July, 2008; revised 23 September, 2008)
}

\begin{abstract}
The steady response of the free surface of a fluid in a porous medium is considered during extraction of the fluid through a line sink. A conformal-mapping approach is used to derive exact solutions to a family of problems in which the line sink is placed at the apex of a wedge-shaped impermeable base, including the limiting cases of an unbounded aquifer and a flat-bottomed aquifer of finite depth. Both critical cusp solutions and sub-critical solutions are computed exactly as Fourier sine series.
\end{abstract}

2000 Mathematics subject classification: primary 76S05.

Keywords and phrases: porous medium, selective withdrawal, hodograph method, exact solution, line sink.

\section{Introduction}

Extraction from layers of fluid of different density within a porous medium is important in both groundwater aquifers and oil reservoirs, among other applications. Oil often lies above water and below gas, while fresh and salt water often form into subterranean layers. When fluid is withdrawn from such layers, the extracted fluid comes from the layer surrounding the point of removal unless the pumping rate is high enough to pull the interface between the layers directly into the outlet. If the withdrawal rate is constant below this critical rate and the pressure forces acting on the fluid are in equilibrium with the gravity force, the interface will reach a stable shape above or below the well. The critical flow rate is defined as the maximum rate at which only the layer adjacent to the sink is withdrawn. At a higher "supercritical rate", fluid from both layers will be removed. Yu [11] and Henderson et al. [5] computed solutions of this type, and more recently Hocking and Zhang [6] found a range of such supercritical solutions using boundary element methods.

\footnotetext{
${ }^{1}$ Mathematics and Statistics, Murdoch University, Western Australia 6150, Australia; e-mail: g.hocking@murdoch.edu.au.

${ }^{2}$ School of Engineering, Gold Coast Campus, Griffith University, Queensland 4222, Australia.

(C) Australian Mathematical Society 2009, Serial-fee code 1446-1811/09 \$16.00
} 


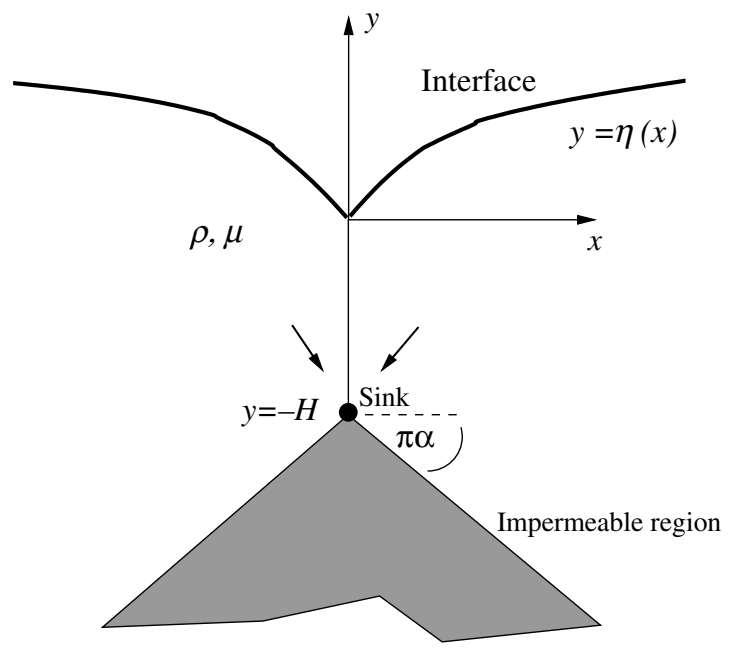

FIGURE 1. Schematic of the flow into a link sink from a single layer above an impermeable wedge-shaped region.

Since the work of Muskat and Wyckoff [9], many scientists have studied critical withdrawal by using analytical methods for various aquifer configurations; see for example [3, 4, 7, 8, 12, 13]. Typically, the hodograph method can be used to solve exactly for the critical flow in which a cusp forms on the interface above (or below) the outlet. Bear and Dagan [3] computed the critical single-layer flow in a range of cases using the hodograph method, while McCarthy [7] computed solutions for extraction from the upper, lighter layer in which the line sink (extraction point) was situated below a horizontal, impermeable base and above the interface.

In all of this work in two dimensions, the logarithmic nature of the singularity due to the line sink leads to the interface levelling off at an infinite depth above or below the line sink. This unrealistic situation was resolved by McCarthy [8] by assuming a constant potential zone a long way from the sink, the so-called "lateral edge drive" model, and by Zhang and Hocking [12] by assuming the interface met a horizontal, impermeable boundary at some distance from the sink. These two corrections lead to very similar results.

In this note, exact solutions (as a Fourier series) are computed for the shape of the interface in both subcritical and critical situations for the geometry as shown in Figure 1, in which the outlet (a line sink) is placed at the apex of an impermeable wedge-shaped region. The method is a variation on the series method used by McCarthy [7] but goes more directly to the required mapping and is extended to consider subcritical flow situations. Series coefficients can be computed exactly as integrals.

Solutions can be found at any value of $\alpha \in[0,1 / 2]$ and for any flow rate at or beneath the critical value. The critical flow rates agree exactly with those obtained using the hodograph method. A nice feature of the method is that it can 
be used for any geometry in which the impermeable region can be described as a set of linear segments, in a manner similar to using conformal mappings via the Schwartz-Christoffel transformation. Another nice feature of the solution is that once the Fourier coefficients have been obtained for a particular bottom angle $\alpha$, the solution for all flow rates is available without new calculations.

Results show that there exist solutions for every withdrawal rate up to the critical value at which a cusp forms on the free surface, and that as the angle $\alpha$ increases, that is the wedge becomes narrower, the critical flow rate increases.

\section{Formulation and solution}

2.1. Equations Consider a homogeneous and isotropic porous medium with intrinsic permeability $\kappa$. A fluid of density $\rho$, and dynamic viscosity $\mu$, lies above a wedge-shaped region of impermeable rock with equation $y=B(x)$, as seen in Figure 1. A line sink $(S)$ is located at the top of the wedge at $(0,-H)$. The sink extracts a total flux $Q$ per unit time per unit width. Note that if $\alpha=0$ the base is horizontal, while if $\alpha=1 / 2$, the impermeable region disappears and the fluid is unbounded below.

Using complex variables, let the physical plane correspond to the right half of the $z$-plane, shown in Figure 1, where $z=x+i y$. Let $y=N(x)$ be the equation of the interface. The velocity potential in two-dimensional steady flow satisfies Darcy's Law:

$$
\Phi=\frac{\kappa}{\mu}(p+\rho g y), \quad y<N(x)
$$

where $\kappa$ is the intrinsic permeability, $p$ is the pressure at the location of $y$ and $g$ is gravitational acceleration. The pressure on the interface between the two immiscible fluids must be constant if we assume the fluid in the upper region to be stagnant. Without loss of generality, this constant can be set to zero, that is $p=0$ on $y=N(x)$, so that

$$
\Phi(x, N(x))=K N(x) \quad \text { where } \quad K=\frac{\kappa g \rho}{\mu}
$$

is the hydraulic conductivity. Defining a velocity potential $\Phi(x, y)$ such that the seepage velocity $\mathbf{q}=-\nabla \Phi$, and combining this equation with the continuity equation, $\operatorname{div} \mathbf{q}=0$, leads to the requirement that $\Phi$ must satisfy Laplace's equation:

$$
\nabla^{2} \Phi(x, y)=0, \quad y<N(x) .
$$

A major benefit of this formulation in complex variables is that any analytic function satisfies Laplace's equation, so that we may define a complex potential function, $F(z)=\Phi+i \Psi$, and so long as this is analytic, it will only remain to satisfy the conditions on the boundaries. $\Psi(x, y)$ can be thought of as a stream function and must be constant along the interface and bottom boundary.

In order to model the extraction we employ a line sink, so that the complex potential satisfies

$$
F \rightarrow \frac{Q}{\pi(\alpha+1 / 2)} \ln (z+i H) \quad \text { as } \quad z \rightarrow-i H,
$$


and $Q$ is therefore the total dimensional flux from the region. The fluid cannot flow through the impermeable base and so we implement the condition

$$
\Phi_{y}=-(\tan \pi \alpha) \Phi_{x} \quad \text { on } \quad y=B(x)=-H-x \tan \pi \alpha .
$$

Notice that the potential due to the sink is logarithmic and so the condition on the interface (2.2) leads to a free-surface boundary of unbounded elevation as $x$ approaches infinity. This phenomenon has been known of at least since the work of Muskat and Wyckoff [9] and while it is somewhat unrealistic, it cannot be avoided in this formulation except by imposing some "artificial" horizontal boundary conditions such as in McCarthy [8] or Zhang and Hocking [13].

In what follows we nondimensionalize with respect to the length $H$ and the potential $Q /(\pi(\alpha+1 / 2))$, that is let

$$
x=H \hat{x}, \quad y=H \hat{y}, \quad N=H \eta \quad \text { and } \quad \Phi=\frac{Q}{\pi(\alpha+1 / 2)} \phi
$$

so that (2.2) becomes

$$
\phi=G^{-1} \hat{y} \quad \text { where } \quad G=\frac{K H \pi(\alpha+1 / 2)}{Q}
$$

is the nondimensional withdrawal rate and the potential $\phi$ has the behaviour,

$$
f \rightarrow \ln (\hat{z}+i) \quad \text { as } \quad \hat{z} \rightarrow-i .
$$

The bottom condition (2.5) can now be written as

$$
\phi_{\hat{y}}=-(\tan \pi \alpha) \phi_{\hat{x}} \quad \text { or as } \quad \psi(\hat{x}, \hat{y})=-1 \quad \text { on } \quad \hat{y}=-1-\hat{x} \tan \pi \alpha .
$$

Henceforth, we will drop the hats from the nondimensional variables for convenience.

2.2. Solution method We seek solutions in which the flow into the sink originates from the lower layer only, using a formulation similar to that used by McCarthy [7], and by Tuck and Vanden-Broeck [10] for the analogous surface-water flow problem. The potential function $f=\phi+i \psi$ can be mapped to the lower half of the unit circle using the mapping

$$
e^{f}=4 t(1+t)^{-2}, \quad|t| \leq 1 .
$$

In order to find a solution, we now choose a mapping from the $t$-plane to the $z$-plane according to the relation

$$
\frac{d z}{d t}=-i \frac{t^{\alpha-1 / 2}}{(t+1)^{1+2 \alpha}} \sum_{k=0}^{\infty} b_{k} t_{k},
$$

where the coefficients $b_{k}, k=0,1,2, \ldots$ are real. McCarthy [7, 8] performed a similar step using a variant of the usual hodograph transformation. Under this 

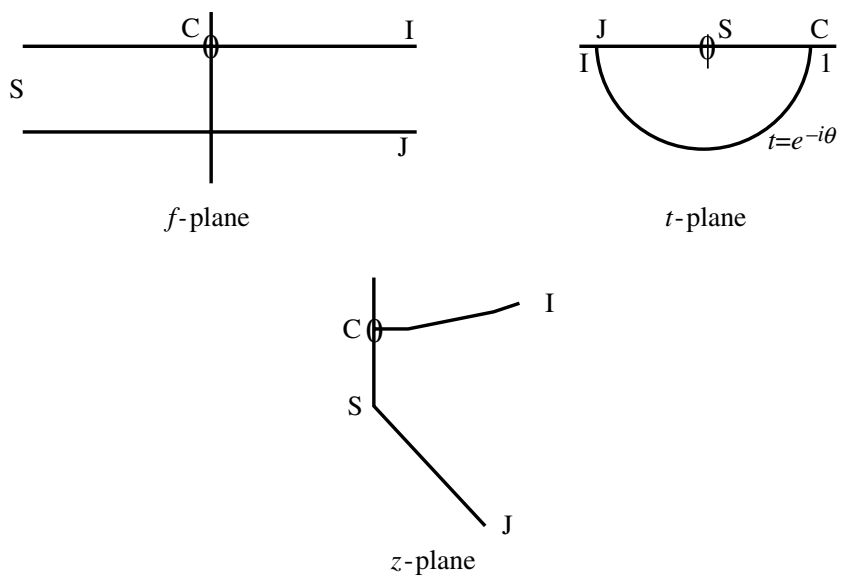

FIGURE 2. The $f, z, t$-planes used in obtaining the solution.

transformation, the line $-1<t<1$ corresponds to the line of symmetry $(x=0$, $-1<y<0)$, and the bottom impermeable boundary to $y=-1-(\tan \pi \alpha) x$. The line sink is situated at $t=0$. The free boundary (interface) runs along the $\operatorname{arc} t=e^{-i \theta}$, $0 \leq \theta<\pi$. This mapping can be obtained directly as shown in Figure 2 or via a mapping through the upper half plane if necessary. The process can easily be adapted to include a range of boundary shapes so long as there are linear line segments along the bottom of the aquifer, using something akin to a Schwartz-Christoffel transformation.

Since the choices made above satisfy all of the conditions on the flow except along the free surface, it remains to satisfy that condition. Noting that if we differentiate (2.2) along the interface with respect to $\theta$, then a modified version is

$$
\frac{d \phi}{d \theta}=G^{-1} \frac{d y}{d \theta} \quad \text { on } \quad t=e^{-i \theta}, \quad 0 \leq \theta<\pi
$$

It immediately follows from differentiating (2.10) that $y^{\prime}(\theta)=G \phi^{\prime}(\theta)=G \tan (\theta / 2)$ and so

$$
y(\theta)=-2 G \log (\cos (\theta / 2))
$$

holds for any angle, $\alpha$, and flow rate $G$. Furthermore, substituting $t=e^{-i \theta}$ it can be shown that (2.12) requires

$$
G \tan \left(\frac{1}{2} \theta\right)\left(2 \cos \left(\frac{1}{2} \theta\right)\right)^{1+2 \alpha}=\sum_{k=1}^{\infty} b_{k} \sin k \theta, \quad 0 \leq \theta<\pi
$$


The problem has now reduced to finding a Fourier sine series representation for the function on the left side of this expression, and the solution for the series coefficients is

$$
b_{k}=\frac{2 G}{\pi} \int_{0}^{\pi} \tan \left(\frac{1}{2} \theta\right)\left(2 \cos \left(\frac{1}{2} \theta\right)\right)^{1+2 \alpha} \sin k \theta d \theta, \quad k=1,2,3, \ldots
$$

We note that $b_{0}$ is not determined at this stage. However, this is fortuitous because $b_{0}$ determines the value of the non-dimensional withdrawal rate, $G$, and hence once we know $b_{k}, k=1,2,3, \ldots$, it is only the value of $b_{0}$ that changes for any given value of bottom angle $\alpha$. The value of $b_{0}$ can be obtained using the fact that we know that the distance from $S$ to $C$, that is the sink to the surface, must be unity, so that integrating (2.11) along the real $t$-axis from $t=0$ to $t=1$ gives

$$
G^{-1}=\left|a_{0} \int_{0}^{1} \frac{t^{\alpha-1 / 2}}{(1+t)^{1+2 \alpha}} d t+\sum_{k=1}^{\infty} a_{k} \int_{0}^{1} \frac{t^{k+\alpha-1 / 2}}{(1+t)^{1+2 \alpha}} d t\right|
$$

after making the simple adjustment, $b_{k}=G a_{k}, k=0,1,2, \ldots$ In fact all of these integrals can be evaluated "exactly" as a series using hypergeometric functions [1], and since they are not singular it is easy to compute them numerically.

In all cases, the critical flows have a cusp in the interface directly above the sink. In order for this to be the case, the condition

$$
\sum_{k=0}^{\infty} b_{k}=0
$$

must be satisfied, since otherwise the speed of the fluid at this point would be zero, that is, the point is a stagnation point of the flow. Therefore, $b_{0}$ and hence $G_{\text {crit }}$ can be found once $b_{j}, j=1,2,3, \ldots$ are known, since $b_{0}=-\sum_{k=1}^{\infty} b_{k}$ from (2.17).

Finally, the shape of the free surface can be obtained by integrating (2.11) along the line $t=e^{-i \theta}$ to obtain the value of $x(\theta)$ corresponding to (2.13), giving

$$
x(\theta)=G \sum_{k=0}^{\infty} a_{k} \int_{0}^{\theta} \frac{\cos k \theta}{(2 \cos (\theta / 2))^{1+2 \alpha}} d \theta .
$$

Notice that only the expression for $x(\theta)$ includes $a_{0}=b_{0} / G$, and it is this that determines the different shapes of the free surface as $G$ changes. At the critical value, this gives a cusp shape through Equation (2.17), while for lower values of $G$ a corner forms representing a stagnation-point flow.

\section{Results}

Figure 3 shows several solutions with different values of $b_{0}$ for the case $\alpha=0$, that is, the sink lies on a horizontal impermeable base, corresponding to nondimensional withdrawal rate values of $G=0.7214$ (critical), 0.4 and 0.24 . Figure 4 shows 


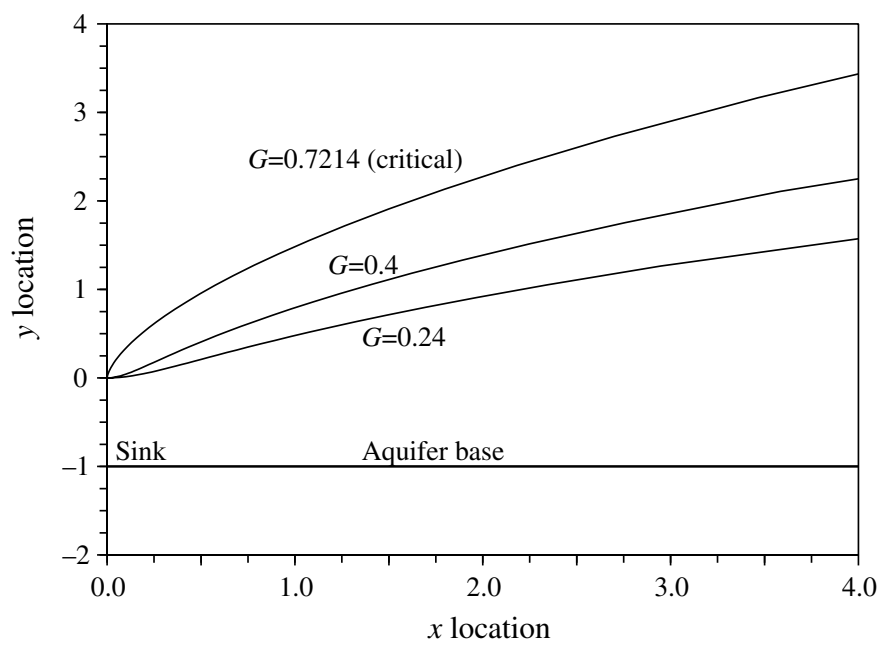

FIGURE 3. Free surface shapes with $\alpha=0$, that is horizontal base, for $G=0.7214$ (critical), 0.4 and 0.24. The sink is at $y=-1$ and the impermeable base is indicated.

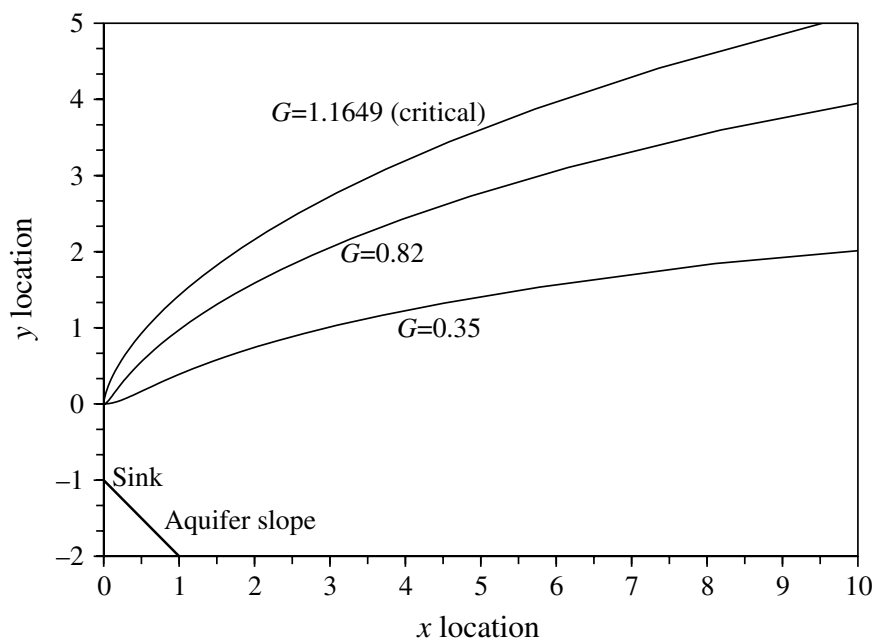

FIGURE 4. Free surface shapes with $\alpha=0.25$ for $G=1.1649$ (critical), 0.82 and 0.35 . The sink is at $y=-1$ and the impermeable base is indicated. 
similar solutions with a 45-degree angle downslope beneath the sink, for $G=1.1649$ (critical), 0.82 and 0.35 .

These results show that as $G$ increases, the free surface steepens above the sink until a critical value is reached at which the stagnation-point solution suddenly draws down into a cusp shape. When $G$ is zero, that is no flow, the free surface is simply horizontal at $y=0$.

It is to be expected that there may be special cases in which the integrals can be evaluated explicitly, and the simplest is the case with $\alpha=1 / 2$, since in that case Equation (2.14) reduces to simply $2 G \sin \theta=b_{1} \sin \theta$ or $G=b_{1} / 2$ and $b_{2}=b_{3}=$ $\cdots=0$. Applying (2.17) gives that $b_{0}=-b_{1}=-2 G_{\text {crit }}$, and combining with (2.16) this means that $G_{\text {crit }}(\alpha=1 / 2)=(2-2 \ln 2)^{-1}$, as in Bear [2]. However, larger values of $b_{0}$ will provide the solution for smaller values of $G$ for this flow, similar to those shown in Figures 3 and 4. Again, once the values of $b_{k}, k=1,2, \ldots$ are known, $b_{0}$ is determined by the choice of $G$. In the formulation given here, the surface shape for the critical flow can be written as

$$
x(\theta)=2 G\left[\tan \left(\frac{1}{2} \theta\right)-\frac{1}{2} \theta\right], \quad y(\theta)=-2 G\left[\log \left(\cos \left(\frac{1}{2} \theta\right)\right)\right] .
$$

Another case that can be simplified is $\alpha=0$, that is the case in which the sink lies on a flat horizontal base, but in this formulation the solution is available only as a series that can be used to check the results of this method.

Critical flows, with a cusp on the surface, can be computed for all of the geometries considered from $\alpha=0$ to $\alpha=0.5$, and these critical values are shown graphically in Figure 5. Comparison with the hodograph solutions given in Bear and Dagan [3] and McCarthy [7] shows that these agree with the values at $\alpha=0,1 / 2$, thus verifying the solutions. The variation in nondimensional withdrawal rate with angle $\alpha$ is almost linear, but is not accounted for simply by the increase in flux values as the angle opens up.

The critical flows can also be computed using the hodograph technique (not shown) for any angle $\alpha$ and the results agree exactly with those obtained using this method.

\section{Conclusions}

The flow into a line sink within a porous medium from an aquifer bounded below by a vertical wedge and above by a free surface has been computed for a range of flow rates up to the critical value using a relatively simple mapping function. The solution appears as a Fourier sine series for which the coefficients can be written exactly as an integral, and once obtained for a single value of bottom angle $\alpha$, all subcritical flows can be immediately computed by varying only the value of $G$.

Imposing a simple condition on the series coefficients also enables us to compute the critical flow for each case, and these were found for a set of angles over the realistic interval, $\alpha=0$ to $\alpha=1 / 2$. These agreed with those in the literature, and showed that as the angle increased from horizontal to vertical, the critical value required to cause coning increased significantly. 


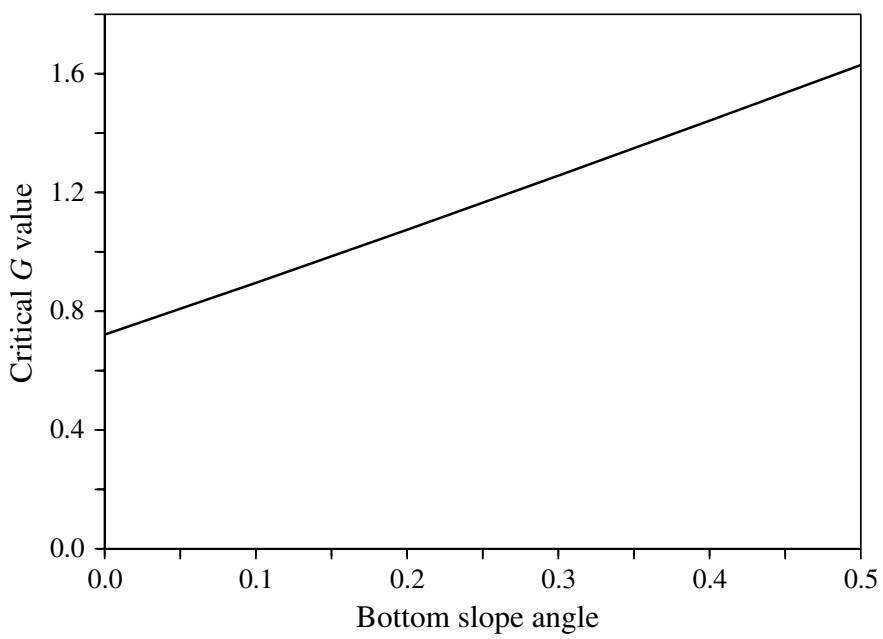

FIGURE 5. Critical values of the nondimensional withdrawal rate, $G_{\text {crit }}$ for different bottom slopes, $\alpha$. $\alpha=0$ corresponds to the sink on a horizontal impermeable base, and $\alpha=1 / 2$ to a sink in a semi-infinite aquifer (unbounded below).

The power of the method is that it can be easily implemented for any bottom shape consisting of linear segments, and that once the critical flow is obtained, all subcritical flows can immediately be computed by simply changing the value of $b_{0}$ and hence $G$. If the value of $G$ exceeds the critical value, then the procedure given in this work is no longer valid and a two-layer solution procedure such as that in Hocking and Zhang [6] would need to be implemented. Work is continuing to obtain these supercritical flows.

\section{References}

[1] M. Abramowitz and I. A. Stegun (eds), Handbook of mathematical functions (Dover, New York, 1970).

[2] J. Bear, Dynamics of fluids in porous media (Dover, New York, 1972).

[3] J. Bear and G. Dagan, "Some exact solutions of interface problems by means of the hodograph method", J. Geophys. Res. 69 (1964) 1563-1572.

[4] F. M. Giger, "Analytic 2-D models of water cresting before breakthrough for horizontal wells", SPE Res. Eng. 4 (1989) 409-416.

[5] N. Henderson, E. Flores, M. Sampaio, L. Freitas and G. M. Platt, "Supercritical fluid flow in porous media: modeling and simulation", Chem. Eng. Sci. 60 (2005) 1797-1808.

[6] G. C. Hocking and H. Zhang, "Supercritical withdrawal from a two-layer fluid through a line sink in a porous medium", J. Eng. Math. (2008) in press.

[7] J. F. McCarthy, "Gas and water cresting towards horizontal wells", J. Austral. Math. Soc. (Series B) 35 (1993) 174-197.

[8] J. F. McCarthy, "Improved model for water cresting", J. Aust. Math. Soc. (Series B) 35 (1993) 207-222.

[9] M. Muskat and R. D. Wyckoff, "An approximate theory of water coning in oil production", Trans. AIME 114 (1935) 144-163.

[10] E. O. Tuck and J.-M. Vanden-Broeck, "A cusp-like free-surface flow due to a submerged source or sink", J. Aust. Math. Soc. (Series B) 25 (1984) 443-450. 
[11] D. Yu, K. Jackson and T. C. Harmon, "Dispersion and diffusion in porous media under supercritical conditions", Chem. Eng. Sci. 54 (1999) 357.

[12] H. Zhang, G. C. Hocking and D. A. Barry, "An analytical solution for critical withdrawal of layered fluid through a line sink in a porous medium”, J. Austral. Math. Soc. (Series B) 39 (1997) 271-279.

[13] H. Zhang and G. C. Hocking, "Axisymmetric flow in an oil reservoir of finite depth caused by a point sink above an oil-water interface”, J. Eng. Math. 32 (1997) 365-376. 